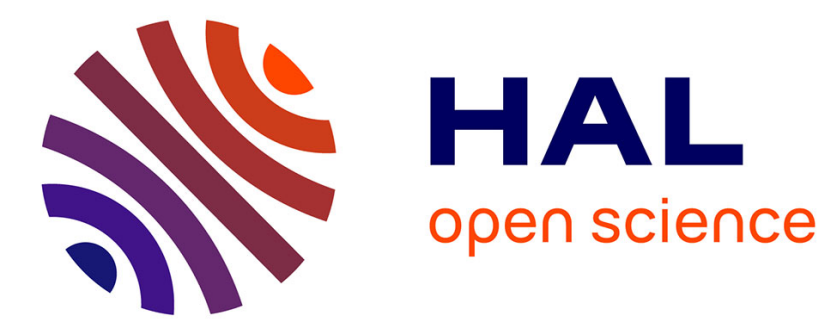

\title{
Les enquêtes de terrain en France
}

Gabriel Bergounioux

\section{To cite this version:}

Gabriel Bergounioux. Les enquêtes de terrain en France. Langue française, 1992, 93 (1), pp.3 - 22. 10.3406/lfr.1992.5808 . halshs-01842502

\section{HAL Id: halshs-01842502 \\ https://shs.hal.science/halshs-01842502}

Submitted on 18 Jul 2018

HAL is a multi-disciplinary open access archive for the deposit and dissemination of scientific research documents, whether they are published or not. The documents may come from teaching and research institutions in France or abroad, or from public or private research centers.
L'archive ouverte pluridisciplinaire HAL, est destinée au dépôt et à la diffusion de documents scientifiques de niveau recherche, publiés ou non, émanant des établissements d'enseignement et de recherche français ou étrangers, des laboratoires publics ou privés. 


\section{Les enquêtes de terrain en France}

Gabriel Bergounioux

\section{Citer ce document / Cite this document :}

Bergounioux Gabriel. Les enquêtes de terrain en France. In: Langue française, n93, 1992. Enquête, corpus et témoin. pp. 322 ;

doi : https://doi.org/10.3406/lfr.1992.5808

https://www.persee.fr/doc/lfr_0023-8368_1992_num_93_1_5808

Fichier pdf généré le 05/05/2018 
Gabriel BERGOUNIOUX

Université d'Orléans

\title{
LES ENQUÊTES DE TERRAIN EN FRANCE
}

\author{
"On peut reproduire des mots que dit la voix; \\ (...) \\ Il reste le souvenir dit la voix (elle pense \\ peut-être à demain) » \\ James Sacré : Viens, dit quelqu'un
}

Ce numéro fait état de recherches; il esquisse des bilans : où en sont aujourd'hui les pratiques d'enquêtes en France, qu'il s'agisse d'étudier des patoisants ou des migrants, la variation entre classes d'âge ou entre classes sociales - mais qui parle encore de classes sociales? Pour saisir à la fois la logique à l'œuvre dans le travail de terrain et le rapport des enquêteurs à leur terrain - et aux autres enquêteurs - on a privilégié le retour à des enquêtes qui, en leur temps, ont revêtu une signification exemplaire, et ce retour a été accompli par des linguistes qui avaient affronté le même terrain et le terrain même dans le Centre-Ouest de la France, opérant de nouveau sur ces zones déjà parcourues, cartographiées, répertoriées.

Ce retour conduit à d'autres interrogations qu'à l'évaluation des différences mises en perspective. Il ne s'agit pas de se démarquer, ou pire d'établir un sottisier, mais de réfléchir sur les conditions qui ont transformé notre approche, nos conceptions du travail avant d'en revenir au constat formulé par Claude Lévi-Strauss: comme l'ethonologue, le linguiste de terrain est perpétuellement confronté à l'écrasement progressif des données, à la disparition de son objet ${ }^{\prime}$ alors qu'à document égal, il se sent apte à déchiffrer toujours plus du réel, comme si, au cours des temps, l'affinement des savoirs devait compenser l'érosion des spécificités observables. Au fond, la question peut se formuler crûment : en quoi serions-nous devenus plus savants? Qu'avons-nous appris de la confron-

1. Un problème crucial pour les dialectes du français mais aussi dans toute étude de phénomènes non répertoriés par l'écrit : les données apparaissent toujours instables, labiles, évanescentes, et finalement fragmentaires, à l'enquêteur. 
tation aux locuteurs? Et des théories linguistiques actuelles ? À l'inverse, y a-t-il trace, dans ces théories linguistiques, des enquêtes, des corpus ${ }^{2}$ ?

\section{Vie et mort de la sociolinguistique en France}

Ce numéro a une ambition. Il prend acte du recul généralisé du travail de terrain et des disciplines qui s'en revendiquaient, notamment de la sociolinguistique qui, à la différence de la dialectologie, est une aventure strictement contemporaine ${ }^{3}$ : en 1967 , dans deux numéros doubles de la Revue de l'Enseignement supérieur, l'article « Linguistique et sociologie » d'O. Burgelin se contente d'établir un parallèle entre les travaux des uns (Martinet, Benveniste, Hjelmslev, Troubetzkoy) et des autres (Parsons, Lévi-Strauss) avant de conclure sur un rapprochement qui tarde à venir :

« Le nombre des problèmes communs à la linguistique et à la sociologie, l'étendue de leurs points de jonction, leurs parentés épistémologiques, les implications qu'elles ont l'une et l'autre sur d'autres disciplines, tout indique la fécondité de rapprochements entre elles. " ${ }^{4}$

Et dans l'article sur « le français », Robert-Léon Wagner déclare :

« Mais cela ne veut pas dire que sur d'autres domaines la linguistique française n'appelle d'autres vocations et ne traverse une période de profonds renouvellements. Ainsi une société est une hiérarchie. Le français commun propose, à ceux qui en occupent les différents étages, un moyen de s'entendre et de communiquer sur des choses essentielles. Mais, dans l'usage, le français se diversifie perpendiculairement suivant les niveaux que constituent les couches sociales. (...) Voilà un domaine qui n'a été jusqu'ici prospecté que par quelques chercheurs, au hasard d'enquêtes fragmentaires. " 5

En note, Wagner ne trouve à citer que Damourette et Pichon, F. Brunot (La pensée et la langue) et Marcel Cohen pour ses Regards sur la langue française, sans mentionner Pour une sociologie du langage paru onze ans plus tôt.

2. Sur cette question, le numéro 21 des Travaux de linguistique (1990) a apporté un certain nombre de réponses.

3. On peut mentionner la collaboration de Meillet au groupe durkheimien : on serait en peine d'en marquer l'incidence dans l'avènement d'une linguistique sociale. Au demeurant, ni la sociologie durkheimienne, ni la linguistique de Meillet ne sont des pratiques de terrain.

4. Burgelin (1967), pp. 87-88.

5. Wagner (1967), p. 17. 
L'explosion des recherches en sociolinguistique reflète pour une part les bouleversements de 68 dans le champ universitaire ${ }^{6}$. La linguistique, pour des raisons conjoncturelles où la mode et les modèles américains eurent leur part, en a amplifié l'incidence et plus que d'autres en subit le contrecoup ? Dans le même temps, la dialectologie, moins sensible à de telles interférences, ancrée sur son terrain et organisée en marge de l'enseignement supérieur, arrive au terme de ses programmes les plus représentatifs, à commence par les Atlas linguistiques de la France par régions. Aujourd'hui, la seule entreprise d'État concernant la variété des français, corrélée à des facteurs géographiques, s'attache à la francophonie hors de France.

Pourtant, au service de nombreux projets, la sociolinguistique dispose d'une revue, de publications et même de programmes aussi ambitieux que le « Français des années 80 » du CREDIF. L'ethnographie de la communication et la pragmatique ont donné à des notions comme celles d'actes de discours ou de contexte une validité opératoire qui requièrent une analyse sociale. Il n'empêche, la linguistique de terrain, et singulièrement la sociolinguistique, semblent s'essouffler, incapables de rivaliser avec les théories les mieux constituées, ou les plus légitimées par le champ scientifique, la grammaire générative, si décriée, gardant à cet égard un prestige indiscutable. La sociolinguistique s'enseigne encore mais sans autre référence que celles qu'elle s'était données à sa fondation : Bernstein, Bourdieu, Labov pour ne citer que les contemporains, sans non plus progresser de façon significative à partir d'eux ${ }^{8}$. Quant à la formation universitaire, elle peut se réduire, après quelques éléments théoriques, à expédier un étudiant avec un thème, un questionnaire modèle (ou de chaleureux encouragements à l'entretien non directif) et un magnétophone à la rencontre de personnes de son entourage. Une fois épuisée la démarche sociologique, quelle part reste acquise à la linguistique?

L'âge d'or de la sociolinguistique en France n'aurait-il duré qu'un quart de siècle, un instant plus éclatant d'avoir rassemblé au milieu de son âge, en 1979, les chercheurs qui s'affrontaient pour la définition légitime du champ, à Rouen? Ne faudrait-il y voir qu'une effervescence, une critique sans contenu, quelque intrusion d'un intérêt politique ${ }^{9}$ consti-

6. Cf. Bourdieu (1984).

7. D'autant que l'exercice de la sociolinguistique a été de part en part conflictuel : son histoire, en France, est plutôt une suite de querelles sur son statut et ses définitions qu'une succession d'enquêtes et de constructions d'objet.

8. D'autres théories, celles de Weinreich ou de Fishman par exemple, ont eu en France une incidence plus limitée.

9. Cf. Gadet (dir.) à paraître. 
tutif de l'engagement des agents dans un choix négatif puisque la sociolinguistique demeure une discipline dominée à l'intérieur de la linguistique? Si l'on suspend ce qui peut être la marque d'un retour au réel de la parole auquel manquent des théories dont l'entreprise d'objectivation suppose le refoulement de la variation, que reste-t-il ?

Non sans esprit de polémique, on pourrait établir quelque parallèle entre la situation des premiers patoisants (entendant, par là, ces amateurs qui consacrèrent de leur temps à l'étude des patois) et celle des premiers sociolinguistes. Comme certains nobles déclassés, au $\mathrm{XIX}^{\mathrm{e}}$, avaient $\mathrm{pu}$ imaginer la perpétuation et la légitimation de leur position symbolique à travers l'apologie de la culture et des valeurs d'une paysannerie « enchantée ", de même, auprès des nombreux assistants recrutés autour de 68 et en position multiplement dominée (in fine, comme linguistes face aux littéraires et comme assistants face aux professeurs ${ }^{10}$ ), la sociolinguistique pouvait être investie d'une double fonction: critiquer les positions dominantes - universitaire, scientifique et politique - d'où étaient exclus ceux qui les mettaient en cause tout en assurant la défense d'intérêts catégoriels universalisés dans une référence parfaitement mythique à la classe ouvrière. Dans les deux cas, on relèverait la même fascination pour l'adversaire désigné : comme les patoisants attendaient leur reconnaissance des milieux littéraires parisiens qu'ils déclaraient honnir, de même l'anti-américanisme militant pouvait faire bon ménage avec des modèles qui étaient tous anglo-saxons - mais, il est vrai, souvent critiques avec la politique de leur pays d'origine. Là encore, à l'attitude d'une noblesse dominée et dévaluée par un nouveau système de la production et des échanges correspondrait la réaction de jeunes universitaires peu assurés, sauf à établir quelque version nationale de la science, de pouvoir maîtriser les circuits des sciences humaines mondialisées par les États-Unis qui s'assurent, à ce moment, un empire sans partage. Ces remarques, trop rapides, devraient être étayées au moyen d'autres paramètres comme l'évolution des publics étudiants ou l'opposition entre centres universitaires parisiens et provinciaux.

Néanmoins, tout se passe comme si la visée critique d'une linguistique en souffrance de social - qui rappelle comment s'opère le travail d'extraction de la langue hors des discours, quel en est le coût requérait la réaction de défense d'un groupe précarisé par la redéfinition des fonctions et des statuts du champ dans lequel il est inscrit. La nécessité de ce rappel peut varier : plus les procédures sont abstraites,

10. Il faudrait tenir compte aussi du rôle du marxisme, à la fois comme option politique immanente à un cursus universitaire - cf. Bourdieu / Passeron (1964) - et comme relais syndical dans la prise en charge des revendications collectives, à la fois dans leur fond et dans leur forme. 
modélisables par graphes et algorithmes, moins la déperdition provoquée par le philologue ou le linguiste est flagrante (ce pourrait être une des raisons du prestige de la syntaxe dans les études linguistiques). Mais dès que fait retour la question du sens ", la complexité des schémas descriptifs destinés à compenser l'amnésie des opérations de séparation entre les locuteurs et leurs parole s'accroît, comme si les procédures d'analyse se subtilisaient moins du fait de la résistance des données que de l'artificialité de leur construction. L'alternative peut-elle dès lors se réduire à choisir, pour la sociolinguistique, entre une vision politique de la science du langage, ou bien quelque retour du refoulé des grammaires formelles?

Ce numéro a une autre ambition, on l'a dit: rappeler que le renouvellement continu de la dimension sociale de la linguistique - qui ne se réduit d'ailleurs pas à la sociolinguistique - n'est pas un épiphénomène lié, par exemple, aux aléas du recrutement dans l'enseignement supérieur; qu'on peut reconstruire, au croisement des théories et des enquêtes, une logique proprement scientifique de ses émergences. Les articles rassemblés dans ce numéro voudraient en porter le témoignage, qu'il s'agisse :

- de l'enquête dialectologique, de ses débuts, au XIX ${ }^{\mathrm{e}}$ siècle, à nos jours, éprouvée sur un terrain circonscrit ;

- des prémices de la sociolinguistique en France avec l'Étude Socio-Linguistique sur Orléans (ESLO) [1966-1970] ;

- de l'état présent des études, dans la confrontation des pratiques, exposées dans la table ronde;

- ou d'un bilan des travaux évalué à partir des collections de la Phonothèque Nationale.

Le même propos aurait pu être illustré d'un autre point de vue par un parallèle entre les écoles linguistiques allemande ou américaine et l'école linguistique française : disons qu'à la méthode comparative, nous avons préféré la méthode historique.

Dans les limites de cette présentation, trois thèmes nous ont retenus :

- Pourquoi, à un certain moment, les attestations des livres, les productions de ses pairs et même son propre sentiment linguistique peuvent apparaître insuffisants ? Autrement dit, "l'enquête ".

- Comment s'effectue alors le choix de représentants à même de porter un témoignage valide sur les usages linguistiques que l'on souhaite

11. La dimension acoustique pose moins de problèmes, depuis longtemps concédée à la phonétique (vs phonologie), qui a reçu sa part congrue; mais pourquoi si peu d'études de sémantique de terrain? 
étudier ? C'est la question des critères et de la forme du mandat accordé à un locuteur particulier, celle qu'on résume dans l'appellation de " témoins ".

- Comment préserve-t-on cette parole ? De quels outils dispose-ton ? Comment est-elle conservée, cataloguée, rendue accessible ? Bref, que désigne-t-on par " corpus»?

Reste l'usage de ces travaux : l'histoire de la linguistique est-elle cumulative? Quelles sont ses procédures de vérification, de discussion ou de réfutation?

\section{Le recours à l'enquête}

À suivre l'histoire des enquêtes en France, on est frappé d'abord par leur discontinuité : on reconstruit des enchaînements, non des filiations. À cela une raison essentielle : le plus souvent, ceux qui s'affrontèrent au terrain ne disposaient pas d'une situation universitaire suffisamment établie pour transmettre à des disciples l'héritage de leur magistère ou de leur charge. Les enquêteurs pouvaient reprendre les études de leurs devanciers mais l'institution à laquelle ils appartenaient était rarement celle de leurs prédécesseurs, et de leurs successeurs. Encore fallut-il la fondation d'institutions qui puissent héberger des personnels dont le service ne s'accomplissait pas devant des étudiants.

\subsection{L'étude des patois (1815-1885)}

Après les enquêtes par correspondance de la Révolution et de l'Empire, une série d'investigations locales fut conduite régionalement par des érudits du cru. Leur âge d'or correspond à la période 1815-1870, avec un objectif, conforme à celui de la Société des Antiquaires de France : recueillir le patois avant que l'unification linguistique ne le fasse disparaître. L'absence de méthode, les hypothèses aberrantes - les « origines celtiques » en constituant le fleuron — sont en partie compensées par la connivence, voire la familiarité avec les locuteurs; la pratique des « us et coutumes 》- expression à la mode au XIX ${ }^{e}$ - et la connaissance des formes d'échanges économiques et sociaux, une certaine sensibilité aux représentations ritualisées de la culture populaire, donnent à la collecte une dimension ethnologique spontanée dont la linguistique devra, dans un premier temps, assumer la perte.

La démarche de ces amateurs de « vieux langage » n'est pas séparable de leur déclassement économique. L'unification du marché des capitaux 
économique et symbolique, en dépréciant le statut des « hobereaux ${ }^{12}$, les induit, en réaction, à revendiquer leur disqualification, privilégiant le capital symbolique - leur parler - contre le capital économique, et le passé de leur classe au dépens de l'avenir. Mais le rapport aux locuteurs reste souvent obscur; le spectacle de Mistral enquêtant à Maguelonne a fait florès ${ }^{13}$ sans qu'on en sache beaucoup plus sur l'élaboration du Tresor dou Felibrige, et moins encore sur les autres amateurs de patois. On doit conjecturer que le sentiment linguistique et l'interrogatoire des employés de maison et des fermiers fut le plus ordinaire recours.

Autour de 1870, la sollicitude du philologue rejoint celle du patoisant : la Société pour l'Étude des Langues Romanes offre une caution savante aux Félibres, de jeunes chartistes s'associent à des amateurs éclairés sous la présidence de Burgaud des Marets pour fonder une association sur le modèle de l'English Dialect Society, le comparatisme romaniste et celtisant justifie un intérêt «objectivé » pour ces parlers. La professionnalisation du marché de la linguistique et la question d'une frontière linguistique oc-oil partitionnant la France, en révélant de plus profonds clivages entre les écoles, mettront un terme précoce à ces convergences.

En séparant locuteur et descripteur, celui-ci se définissant de manière suffisante d'être un savant, la romanistique impose définitivement le recours à l'enquête. Il ne s'agit plus de sauver un patois de la disparition mais d'invalider ou de justifier des thèses dont les attendus et la fonction procèdent d'une théorie du langage inaccessible à des patoisants, indifférente aux enjeux locaux et, en dernière instance, dominante sur les faits. L'imposition de la désignation "gallo-roman », qui escamote la frontière oc / oil, en est un exemple, comme le recours à des isoglosses dont les tracés, fluctuants et foisonnants, autorisent tous les refus de partition en ensembles dialectaux. Antoine Thomas peut être considéré comme le

12. Même le terme manque d'efficacitế : «On donne le nom de hobereau aux petits seigneurs qui tyrannisent leurs paysans" dit Buffon cité par Robert ; la différence est patente avec les équivalents allemand - junker -, italien - latifundiaire - et anglais - gentleman farmer, landlord. La Révolution, en émiettant les grandes tenures foncières nobles, a confirmé le primat de la petite propriété paysanne. Après Oscar Bloch (1935), Pop parle à son tour d'une génération des « Comtes » pour la première génération de ces férus de patois : Jaubert, Chambure, Montesson. La centralisation royale puis jacobine avait déjà laminé la revendication régionale, ce qui conforte la singularité dialectale de la France par rapport à l'Allemagne, l'Italie ou l'Empire habsbourgeois.

13. “ Ce n'est pas dans les vieux livres provençaux qu'il (Mistral) a fait ses recherches : c'est dans l'usage du peuple (...) Je le vis à Maguelonne s'enquérir auprès des pêcheurs pour son grand dictionnaire. Il était là, assis dans le bateau, maniant en connaisseur chacun des agrès, touchant chacune des parties du petit bâtiment et disant : "Nous autres, chez moi, nous appelons cela ainsi, et vous ?" Et les pêcheurs, riants et émerveillés, lui disaient tout leur vocabulaire et il inscrivait ce qui lui était nouveau. Partout, avec les artisans, avec les laboureurs, avec les pâtres, il faisait la même enquête familière et méthodique (...) n, G. Paris in Devoluy (1941), pp. 197-198. Wartburg voit plutôt dans le Tresor une entreprise livresque de seconde main assez peu fiable. 
premier de ces romanistes à avoir accompli une rupture symbolique ${ }^{14}$ avec les témoins en recourant à une notation entièrement phonétique des réponses des locuteurs. La nécessité proprement scientifique du procédé de transcription abolit la continuité entre locuteur et enquêteur d'une part, universitaire et savant non professionnel d'autre part; l'enseignement de Gilliéron à l'École Pratique des Hautes Études, à partir de 1883, en impose le principe : le dialectologue n'a plus à être un dialectophone.

\subsection{ALF et API}

Forts de leur visibilité institutionnelle, à l'Institut Catholique pour l'un, à l'EPHE pour l'autre, Gilliéron et l'abbé Rousselot lancent, en 1887, une Revue des patois gallo-romans, proposant, dès le premier numéro, l'alphabet phonétique composé par Rousselot et une nomenclature d'enquête rurale préparée par Gilliéron ${ }^{15}$. Le projet, qui privilégiait l'atomisation des idiomes par une série d'études au mot à mot et la spécification des variables phonétiques, avait de quoi décourager ceux qui, de défendre leur parler, en avaient au contraire une vision globale, orale, affective. La revue se survit sans revivre dans la Société des Parlers de France dont le Bulletin battit des records de confidentialité.

L'appel d'Alès de Gaston Paris (1888) ${ }^{16}$ n'aura pu suffire à trouver des enquêteurs bénévoles. De s'adresser à des agents dont la légitimité avait été conquise contre leurs origines patoisantes par une qualification en français, sa portée fut infime. Le même projet, renouvelé par Rousselot auprès du clergé, ne lui fournit que quelques élèves. Les propos de G. Paris et de ses élèves reflètent surtout les exigences et les présupposés des linguistes : nécessité d'un maillage serré - l'idée d'un point d'enquête par commune est latente - pour relever des variations qu'on annonce aussi nombreuses que capricieuses; importance du témoignage des patois non pour eux-mêmes mais pour la connaissance du français ; enfin, imminence de leur disparition. On pourrait y retrouver aussi l'erreur d'appréciation des romanistes parisiens concernant leurs capacités de mobilisation symbolique.

Quand, au même moment, Passy grave sur rouleau le premier échantillon du français commun pour en fixer la forme phonétique

14. Son père, entrepreneur en bâtiment du Limousin, est « monté »à Paris. Antoine Thomas grandit dans un univers bilingue français / patois. Peu sûr de son identité vernaculaire, sans tradition intellectuelle familiale, c'est un déraciné, partagé entre l'amour du pays natal et le prestige de la science. Au demeurant, il se détournera assez vite de la dialectologie pour chercher une reconnaissance dans la fondation de la « philologie française n- étude historique d'unités lexicales d'origine ou de sens obscurs.

15. À la même époque, Léon Clédat fonde une Revue des patois qui sera un demi-échec.

16. Invité à prendre la parole devant les représentants des sociétés savantes, G. Paris en profite pour lancer un appel à la collecte des patois. 
authentique de manière instrumentale, avant de reproduire l'expérience sur d'autres langues, c'est un autre projet qui se dessine : celui de l'Alphabet Phonétique International, l'API ${ }^{17}$. Amour du terroir contre cosmopolitisme, inventaire des particularismes locaux contre représentation universelle, la linguistique de l'oral en France se sépare en deux écoles qui ne se confondront plus : la dialectologie, centrée sur le domaine roman, d'une part, et la description des réalisations du français, comparé aux autres langues, d'autre part. La division des études linguistiques entre deux alphabets phonétiques, entre deux types d'institution (la dialectologie ne sera jamais reconnue comme un enseignement à part entière dans les facultés), deux visions du substrat de la variation - cartographie ou classes sociales - reflète jusqu'à nos jours cette dichotomie. Mais alors que l'initiative de Passy se résorbait dans une phonologie contrastive des langues ${ }^{18}$, l' $A L F$ apportait à la dialectologie une visibilité accrue.

Lorsque Gilliéron se résoud à entreprendre, avec de très faibles moyens matériels ${ }^{19}$, l'Atlas linguistique de la France, il procède à des choix symptomatiques : en fond de carte, la France romane moins la Corse ; un maillage déployé en toile d'araignée; un questionnaire rural; l'alphabet de sa Revue. N'ambitionnant qu'une représentativité géographique, il se soucie a minima des positions sociales, comme le montrent les carnets d'enquête d'Edmont. En représentant chaque item sur une carte de France, il annule les locuteurs et désarticule - dé-structure - les parlers, attestés ponctuellement mais non spatialement. Ces «points d'enquête » (par opposition à des « aires d'investigation») sont déterminés par des cercles concentriques, autour d'un barycentre fictif, afin de récuser a priori toute division dialectale. L'exploitation du travail ê̂t dû confirmer les attendus des romanistes parisiens : qu'en dehors du français standard, il n'existait que ces parlers instables, déchirés entre les deux principes que Saussure dénommera « esprit de clocher» et «intercourse ». Ce fut l'inverse qui advint : la séparation oc / oïl était avérée par la superposition des cartes. Désormais, il fallait en revenir à des monographies locales (Millardet, Terracher, Bruneau, Bloch) ${ }^{20}$. Jusqu'à 1945, seuls les enregis-

17. Il n'est pas indifférent à ce projet que Passy soit un militant socialiste, évangéliste de surcroî ; l'API est pour lui plus qu'un résultat de laboratoire, c'est un moyen pour rapprocher les hommes, un outil au service de la paix.

18. L'étude du français populaire ne connut plus d'autre ressource que l'exploitation de sources écrites (Frei) ou l'oreille et la notation au vol (Bauche). On retrouverait la même dominante philologique dans les travaux sur l'argot, depuis leur apparition à la fin du XIX'.

19. Le détail est d'importance ; il fallut l'insistance d'un romaniste membre de l'Académie Française pour obtenir le paiement des frais d'Edmont au plus juste. La science officielle, qui finançait missions de fouilles et publications de l'Institut, rechignait à trouver des crédits pour un atlas constitué sur le terrain.

20. Peu d'études (on pense pourtant à Ronjat) se proposèrent pour fin de déterminer les traits linguistiques spécifiques unifiant une langue régionale. L’objectif des dialectologues français n'était pas de promouvoir un usage légitime des parlers qu'ils étudiaient. 
trements (interrompus en 1914) de Ferdinand Brunot pour les « Archives de la Parole », devenues le Musée de la Parole et du Geste, ambitionnèrent une représentativité nationale. Il n'est d'ailleurs pas interdit de penser que la guerre de 14-18 ait permis à l'initiateur du projet de dénouer une entreprise qui lui pesait et qui l'avait conduit du témoignage linguistique au conservatoire folklorique : après l'enthousiasme des premières expéditions, Brunot ne revint plus sur le terrain. Quand ce travail sera repris, à partir de 1939, par Devigne, la dialectologie laissera place à la collecte du légendaire.

Depuis 45, les principales formes d'enquête semblent correspondre à trois types de motivations :

- les Atlas Linguistiques et Ethnographiques de la France par régions qui tentent de réconcilier l'approche de Gilliéron avec la reconnaissance d'ensembles dialectaux. Initialement présidé par Dauzat, financé par le CNRS, ce programme a été relayé par des monogrpahies dialectologiques plus circonscrites ;

- le « Français Élémentaire », en concurrence avec le BASIC, créé pour promouvoir le français dans des colonies bientôt indépendantes et auprès des techniciens... On peut y rattacher diverses études destinées à l'enseignement du FLE, notamment l'ESLO ;

- les corpus d'application qui, à s'en tenir aux investigations métropolitaines, se répartissent entre la sociolinguistique, la psychodépadogie et la linguistique descriptive ${ }^{21}$.

\section{Le choix du témoin}

La linguistique - comme méthode raisonnée et non triviale de saisie des langues par la transcription phonétique, l'établissement des paradigmes morphologiques et l'inventaire lexicographique - supposait la formation d'un corps de professionnels : le savoir des locuteurs devient factuel par rapport aux principes de description de leur savoir, l'ignorance même participant du statut des témoins. Ils représentent l'unique accès à une connaissance en attente d'objectivation et non de norme ${ }^{22}$, par où s'inaugure la transformation du rapport des linguistes à leur objet, passant du normatif au descriptif, d'abord pour les patois, puis pour le français comme le démontreraient les attestations de Damourette et Pichon. La valeur des résultats n'est plus soumise aux informateurs,

21. Cette répartition peut être vérifiée dans les corpus cités in Jeanjean / Blanche-Benveniste (1987).

22. Où se joue, entre autres, la rupture avec la littérature régionaliste et les académies provinciales. 
incapables d'accéder aux formes de présentation requises par la science, mais aux pairs : le marché de la linguistique conquiert son autonomie en partie dans cette indifférence aux locuteurs.

A l'inverse, dans l'enquête par correspondance, un témoin identifié se mandate lui-même en regard d'une demande qui lui est, généralement, extérieure. Après l'enquête administrée à soi-même - dont le chomskysme constituerait l'ultime avatar - et aux proches, les premières enquêtes fondées sur des témoins anonymes mais géographiquement assignés, dans le scrupule des formes sonores, n'advinrent qu'avec les débats sur la ligne oc/oil, quand l'enquête privilégia l'argumentation interne aux débats philologiques plutôt que la recension administrative ${ }^{23}$. La transition entre l'enquête familière et l'enquête ethnographique pourrait être représentée par Rousselot qui, après avoir échoué dans la détermination de cette frontière linguistique, se replia sur une investigation dans son propre village, en raisonnant à partir des différences notées empiriquement. Edmont, parce qu'il partait à la rencontre d'inconnus, systématisa, à la demande de Gilliéron, l'usage de fiches signalétiques distinctes pour chaque témoin.

Pressé par le temps, Edmont fut obligé de transiger avec les exigences initiales concernant la représentativité réelle de son interlocuteur. Il est plus facile de convenir du choix d'un témoin que de s'accorder sur ce qu'il représente : une région, un canton, une commune ? Les locuteurs de son âge, ceux de sa profession ? Chercher à préciser la validité du témoignage rapproche et oppose la dialectologie et la sociologie, celle-ci visant par principe à multiplier les critères de variation abstraction faite de la variation géographique, celle-là visant à spécifier l'extension topographique du témoignage en abolissant les particularités sociales du témoin. La réduction constante de locuteurs patoisants a probablement contribué à cet élargissement alors que les bouleversements économiques et techniques, l'immigration, la transformation des instances de reproduction contraignaient la sociolinguistique à complexifier ses paramètres. Paradoxalement, le locuteur idéal d'une communauté linguistique homogène n'est peut-être pas très loin de l'idéal du dialectologue.

23. Ces moments de l’investigation dialectologique sont développés dans les articles infra. À noter que l'enquête par correspondance n'en continua pas moins : cf. in Wartburg et al. (1969), à propos de l'enquête de Íéon Clédat. 


\section{La conservation de la parole}

En France, les collecteurs de parole ont « naturellement » eu recours à l'alphabet latin en dépit de son inadaptation aux langues romanes et des errements de l'orthographe. Des systèmes sténographiques en usage depuis la fin du XVIII ${ }^{e}$, nous n'avons pas trouvé d'emploi, dans aucune enquête linguistique. Il est vrai que le descripteur-locuteur ne requérait qu'un aide-mémoire dans ses notations et une convention immédiatement lisible pour ses publications, en se laissant guider par les graphies des mots français ${ }^{24}$. La nécessité d'une notation phonétique est inséparable des lois de la grammaire historique, les premières transcriptions se suffisant d'un compromis entre les trois principes d'une orthographe : l'étymologie, les paradigmes et la phonétique. C'est ainsi que procédèrent poètes bretonnants et félibres, aboutissant à des transcriptions aussi peu satisfaisantes pour l'occitan que pour le breton.

Une approche scientifique, en rupture avec la tradition littéraire, impliquait l'établissement d'un minimum de règles d'équivalence entre l'acoustique et la scription - comme procédèrent Bringuier et Tourtoulon afin d'éviter les ambiguïtés de l'orthographe - et la transcription de marques supra-segmentales ${ }^{25}$. Dans la rivalité entre romanistes parisiens et provençalistes, la rupture accomplie par $A$. Thomas revenait d'abord à accorder le primat à l'enquêteur sur le locuteur, mais au-delà au linguiste sur l'enquêteur. Pour Gilliéron, à Paris, il suffisait que son enquêteur, Edmont, ait « une oreille », même si celle-ci était faillible (les reproches les plus fréquemment faits à Edmont concerneront les erreurs dues à l'ignorance du parler qu'il étudiait); quant à ses points d'enquête, ils dépendaient exclusivement des nécesssités du maillage et non de l'assurance de trouver des informateurs fiables.

Brunot a pu imaginer de supprimer l'intercession de l'enquêteur par ses enregistrements sur disques. De cette conviction, on retiendra l'intuition pionnière, la collaboration avec les professionnels du son, les contraintes techniques - deux fois trois minutes d'enregistrement par disque et la présence encombrante du cornet - et l'exaltation d'une aventure neuve. On retiendra surtout le souhait de ne pas enregistrer seulement des dialectes mais aussi du français parlé - c'est-à-dire populaire - et d'autres langues. Il n'est pas exagéré de dire que la situation ne connut pas de changement qualitatif jusqu'à la diffusion du magnétophone, et encore !

24. Il ne semble pas avoir existé, en France, depuis deux siècles, de descripteur d'une langue régionale qui n'ait lu, parlé et écrit parfaitement le français pour l'avoir appris à l'école.

25. Spécialement pour la notation de l'accent d'intensité, central dans la théorie de l'évolution phonétique et du changement. 
Mais les contraintes de conservation changeaient : tant qu'on en reste à des alphabets, le livre peut servir de véhicule en dépit des problèmes posés par la fonte de caractères phonétiques - l'Atlas linguistique de la France fut un gouffre pour l'imprimeur, Protat, et pour l'éditeur, Champion. Les disques, au contraire, supposaient une organisation différente de la conservation, confiée à l'Institut de Phonétique et au Musée de la Parole et du Geste.

\section{Catalogue, publications, archives}

\subsection{Permanence du témoin}

À partir de quel moment y a-t-il eu souci de garder la trace d'une identification du témoin? Il a fallu que la science se fasse méfiance, soucieuse de contrôler les affirmations; mais aussi que le patois n'apparaisse plus comme la création collective d'un peuple indifférencié comme, dans la vision romantique, la littérature populaire - mais comme le produit, situé dans les paramètres de la linguistique diachronique et comparative, d'une situation particulière, un statut professionnel par exemple. Cette reconstruction du rôle du témoin allait de pair avec la naissance des études de folklore qui assignaient aux locuteurs une forme particulière de dignité, mais aussi avec les règles de la méthode sociologique.

Dans les parcours qui tentent de relier des points d'enquête pour aboutir à la détermination d'une frontière ${ }^{26}$, le témoin n'est plus convoqué comme représentant exemplaire d'un parler mais comme preuve d'une rupture, en un point donné - géographique et phonétique - des parlers. Et ce témoin lui-même est éparpillé dans le morcellement des preuves, la rupture n'en finissant pas d'être démultipliée, des traitements phonétiques du latin à l'usage lexical. Lorsque Nizier du Puitspelu - de son vrai nom Clair Tisseur - , publie son dictionnaire (1887-1890), Paul Meyer le reprend dans Romania, refusant une démarche qui postule l'existence d'un dialecte, en l'occurrence celui du Lyonnais, et exigeant la délimitation géographique mot par mot. Cette représentation, centrée sur la spécificité lexicale (étude des aires de chaque terme), est la forme d'enquête et d'analyse exemplifiée par Gilliéron. Une autre école mettait l'accent sur la spécificité de chaque locuteur : c'est le travail en phonétique

26. C. Wartburg et al. (1969), pp. 26-38. 
de Rousselot ${ }^{27}$. Force est de constater que celui-là travaille à distance dans un bureau sur des jeux de fiches - à partir de 1897 - alors que l'autre mène lui-même son enquête, et dans sa propre famille. Ce sont d'autres termes d'une alternative qui divise la linguistique entre une science objectivée du lexique et une science concrète des locuteurs.

En procédant par enregistrements, Brunot ne proposait que de retarder les choix qui président à l'étude et aboutissent à la séparation du langage et du locuteur. À la différence d'une fiche, sur un rouleau ou un disque, la présence physique de la voix continue de s'imposer : quelle que soit l'étude, elle ne peut abstraire la présence perpétuée d'un locuteur entre autres qui demeure, malgré tout, un homme ou une femme, jeune ou âgé, expansif ou timide... Dès que Brunot entreprend sa collecte, il ponctue son travail de considérants presque affectifs sur ses témoins qui font généralement défaut - sauf familiarité immédiate avec le terrain chez ses collègues, et chez lui dès lors qu'il se fait grammairien.

La sociolinguistique retrouve toute la difficulté particulière de la situation d'enquête en déplaçant le problème, focalisé par la dialectologie sur la différence interlinguistique, vers la distance sociale qui sépare le collecteur de ses témoins. Un des symptômes dans la littérature sociolinguistique serait l'idée d'une enquête menée à l'insu de l'informateur, le micro caché. En dernière instance, ce n'est pas de l'enquête linguistique que sont venus la problématique et le progrès de la méthode d'enquête mais des critiques des sciences sociales.

\subsection{La conservation}

Il semble que, dans les débuts, au XIX ${ }^{e}$, on ait cherché des résultats, que la méthode comme chemin n'ait guère été un souci. Si les matériaux originels sont retrouvés, on constate qu'ils ne contiennent presque rien de plus que ce qui a été livré. Culture savante diffusée par le livre et la revue, la linguistique s'impose des enquêtes sans reste en fonction de formes canoniques de présentation, en listes ou en cartes, pour lesquelles sont établis des questionnaires qui leur soient strictement congruents.

En revanche, peu de textes spontanés : si l'on fait abstraction des corpus folkloriques (chansons, proverbes, récits...), il n'y a guère de ce qui, après 1945, apparaîtra comme le propre du linguiste : des transcriptions. Il serait probablement impossible de faire une histoire des marques énon-

27. La lexicologie tend spontanément vers une personnification, à la manière de Darmesteter (1886) : la phonétique, au contraire, reporte la variation sur les sujets parlants, sauf à viser des tracés d'isoglosse : le mot a un territoire, le trait phonétique un tracé, une ligne; ce sont des géométries distinctes. 
ciatives spontanées ou des ponctuants à partir des documents publiés. Les résultats avoués se présentent comme un ensemble de mots, au mieux comme une collection d'exemples, de phrases traduites du français ou saisies au vol, plus tardivement de quelques discussions menées en patois. La sociologie aura été, en ce domaine, plus utile à la sociolinguistique que la linguistique, celle-ci fournissant les hypothèses et les outils, celle-là les méthodes et les techniques.

\section{Marché de la linguistique et usage des enquêtes}

Après avoir repris les trois moments des enquêtes - motivation, exécution, exploitation - on peut s'interroger sur leur devenir. Après un siècle d'enregistrements, deux siècles d'enquête (depuis Grégoire), quel aura été le destin des documents recueillis ?

On constate que le document brut reste en dernière instance le plus transmissible et le plus utilisable. G. Paris le disait déjà en demandant à ses putatifs informateurs de ne pas s'occuper d'étymologie. Quelle que soit l'enquête, sa faiblesse principielle semble résider dans ses attendus. Une enquête de la qualité de l'ALF est close irréversiblement : on peut seulement en commenter les résultats. Même l'enquête d'Orléans (ESLO) pêche par ses présupposés bernsteiniens. Avant 1950, les travaux sur le français parlé, à l'exclusion des français régionaux, ont procédé par dépouillement de documents écrits ${ }^{28}$. Pour une sociolinguistique de terrain, Martinet, enquêtant dans son offlag, représenterait la transition avec la dialectologie comparatiste et la linguistique structurale, même si, pour des raisons déjà énoncées, la sociolinguistique n'acquerra quelque visibilité qu'avec la croissance quantitative de la linguistique universitaire dans laquelle la pyramide des âges - et des statuts - favorisait les prises de position critiques que les événements de 68 radicalisèrent. Dans le même temps, la sollicitation de domaines connexes pour un français vivant - réforme de l'enseignement et propositions de l'AFEF, expansion du Français Langue Etrangère - modifiait le rapport aux études de langue.

Si l'on esquisse un bilan, on s'aperçoit qu'il n'existe que très peu d'enquêtes en circulation réelle. Il est vrai que la demande est faible : il est

28. À l'exception de Passy et Brunot qui ont laissé une dizaine de minutes d'enregistrement. Pierrette Dubuisson rappelait, lors de la table ronde « Pour une Phonothèque en Région Centre » (octobre 1987) que le CNRS n'avait alloué, à ses débuts, que très peu de bandes magnétiques aux enquêteurs des Atlas Linguistiques de la France par régions, exigeant que les transcriptions soient rapides et la réutilisation des bandes systématique. 
si contraignant de travailler sur ses propres matériaux; les documents d'autrui ne présentent qu'une difficulté supplémentaire. D'autant qu'un document, pour être exploitable, doit avoir été transcrit, éventuellement saisi sur ordinateur; sinon, il est plus facile de procéder soi-même à des enregistrements. Or, très peu de corpus sont accessibles sous cette forme. Le résultat s'ensuit : les corpus déterminent des micro-réseaux de communication, souvent réduits à leur seul collecteur. L'enquête est, en France, une pratique pour laquelle il n'existe pas de demande.

Le marché de la linguistique en France, centralisé par le quasimonopole de Paris sur la circulation des biens symboliques et dominé par les études littéraires dans l'organisation universitaire, favorise objectivement la recherche abstraite, universalisante ou formalisante, au détriment des pratiques de recension descriptives, autrement dit, ceux qui travaillent en bibliothèque plutôt que sur le terrain. La présence dans les bureaucraties d'administration de la science, par exemple, est plus favorable à une carrière dans l'enseignement supérieur que l'enquête. Il convient d'ajouter les temps morts, c'est-à-dire invisibles en termes de résultats, de la constitution de corpus, pour les déplacements, la sélection des opérations et des témoins, les contraintes techniques, la saisie, les transcriptions, le catalogage. Dans toute enquête longue, il y a le risque que le linguiste n'en vienne à faire de son moyen une fin jusqu'à parvenir, à force de précautions, à une forme de présentation d'autant plus achevée qu'elle aura renoncé à l'explication.

Dans la division entre gens de terrain et gens du livre, la logique universitaire ne peut que favoriser ceux qui sont les plus respectueux de sa tradition lettrée. Cette tradition philologique, qui favorise la langue morte - hier le latin et le grec, aujourd'hui la langue littéraire - contre la langue vivante, ne se serait pas perpétuée si facilement si elle n'avait rencontré les intérêts bien compris de tous ceux dont la formation s'est accomplie sans terrain et qui, par l'abstraction, désavouent aussi les vérifications pratiques. L'origine sociale des gens de terrain confirmerait leur domination à l'entrée dans l'université et la manière particulière de perpétuer cette soumission en se livrant à des travaux d'enquête. L'institution, assurant sa reproduction par le recrutement presque exclusif d'étudiants en lettres, se pourvoit d'agents prédisposés, par leur formation, à sanctionner et perpétuer cet ordre. Au demeurant, s'il est courant de trouver des linguistes passés de l'enquête à la philologie (Havet, Thomas, Gilliéron, Rousselot, Dauzat...), il n'est aucun exemple d'une carrière inverse.

Voici un bilan : deux siècles d'enquête, vingt-cinq ans de sociolinguistique et après ? En quoi sommes-nous devenus plus savants? Réti- 
cence à normer la langue ? Suspicion sur la validité des témoignages? Souci de garder une trace analogique ? Oui, et aussi cela : dominée par une linguistique qui ne décrit plus rien d'observé, et même, parfois, peut-être, plus rien d'observable, la linguistique de terrain, sous des noms divers, oppose des interrogations et ménage des crises: théorie du discours, réalisme phonologique, construction du sens, statut de la syntaxe de l'oral, autant de questions qu'une théorie classique, fût-elle générative, peine à dénouer. Si l'on reprenait un exercice qui faisait rire hier, vers 68 justement, de commenter les exemples des grammaires pour calculer la valeur des théories qui s'en justifient, on pourrait s'apercevoir en quoi une linguistique de l'oral nous a fait, à défaut d'être plus savants, moins crédules. Pas d'avancée en linguistique sans ce questionnement de l'oral et pas d'oral sans enquête, sans corpus, sans témoin. Si ce numéro constitue un bilan, il ne serait pas mal venu qu'il soit, aussi, un appel.

\section{BIBLIOGRAPHIE}

Antoine Gérald et MARTIn Robert (dir.), 1985 : Histoire de la langue française 1880-1914, Paris, CNRS.

BAL Willy, 1966 : Introduction aux études de linguistique romane, Paris, Didier.

Balibar Renée et Laporte Dominique, 1974: Le français national, Paris, Hachette.

BaLly Charles, 1913 : Le langage et la vie, Genève, Droz.

Bauche Henri, 1920 : Le langage populaire, Paris, Payot.

BergouniouX Gabriel, 1984 : « La science du langage en France de 1870 à 1885 : du marché civil au marché étatique ", in Chevalier / Encrevé (1984), pp. 7-41.

Bernstein Basil, 1975 : Langage et classes sociales, Paris, Minuit.

Bloch Oscar, 1935 : « Lexicologie-Dialectologie », in Dauzat (1935), pp. 139-186.

Bloch Oscar, 1917 : Atlas linguistique des Vosges méridionales, Paris, Champion.

Bourdeu Pierre, 1964 : Les héritiers, Paris, Minuit.

Bourdeu Pierre, 1970 : La reproduction, Paris, Minuit.

Bourdieu Pierre, 1984: Homo Academicus, Paris, Minuit.

Boyer Henri et Peytard Jean (dir.), 1990 : « Les représentants de la langue : approches sociolinguistiques », Langue Française, 85, février 1990.

Branca Sonia, 1982 : « Espace national et découpage dialectal : deux étapes de la construction de la dialectologie au $\mathrm{XIX}^{e}$ siècle $»$, in Trames, Limoges.

BrANCA-RoSoff Sonia, 1990 : « De la culture de la vigne à la cueillette des fleurs des champs. Les noms de la langue dans la dialectologie française du $\mathrm{XIX}^{\mathrm{e}}$ siècle », in Boyer et Peytard (1990).

Bright William (dir.), 1966 : Sociolinguistics, La Haye, Mouton. 
Bruneau Charles, 1914 : Enquête linguistique sur les patois d'Ardennes, Paris, Champion.

Brunot Ferdinand, 1926 : La pensée et la langue, Paris, Masson.

Burgelin Olivier, 1967 : « Linguistique et sociologie », Revue de l'Enseignement Supérieur, 1-2, pp. 80-88.

Calas Marie-France et Brun-Trigaud Guylaine, 1990: L'oral en collections, Paris, AFAS.

CERQUIGlini Bernard, 1989 : Eloge de la variante : histoire critique de la philologie, Paris, Minuit.

Chambure Eugène de, 1878 : Glossaire du Morvan, Paris / Autun.

Chaurand Jacques, 1985 : «L'essor de la dialectologie », in Antoine / Martin, pp. 601-604.

Chevalier Jean-Claude et Encreve Pierre (dir.), 1984: «Vers une histoire sociale de la linguistique », Langue Française, 63, Paris, Larousse, septembre 1984.

Chevalier Jean-Claude, 1985 : «Les grammaires françaises et l'histoire de la langue », in Antoine / Martin (1985), pp. 577-600.

Cohen Marcel, 1950 : Instructions d'Enquête linguistique, Paris, Institut d'Ethnologie ( $2^{e}$ éd.).

Cohen Marcel, 1956 : Pour une sociologie du langage, Paris, Albin Michel.

CoHen Marcel, 1963 : Nouveaux regards sur la langue française, Paris, Editions Sociales.

Damourette Jacques et Pichon Edouard, 1911 -1950 : Des mots à la pensée (Essai de grammaire de la langue française), Paris, d'Artrey, 6 volumes + 1 supplément.

Da Rmesteter Arsène, 1886 : La vie des mots, Paris, Delagrave.

Dauzat Albert, 1906 : Essai de méthodologia linguistique dans le domaine des langues et des patois romans, Paris, Champion.

Dauzat Albert, 1922 : La géographie linguistique, Paris, Flammarion.

Dauzat Albert, 1927 : Les patois : évolution, classification, étude, Paris, Delagrave.

DAuZat Albert (dir.), 1935 : Où en sont les études de français ?, Paris, d'Artrey.

De Certea U Michel, Julia Dominique, Revel Jacques, 1975 : Une politique de la langue, Paris, Gallimard.

Devigne Roger, 1949 : La Phonothèque Nationale, Paris, Phonothèque Nationale.

Devoluy Pierre, 1941 : Mistral et la rédemption d'une langue, Paris, Grasset.

ESNAULT Gaston, 1965 : Dictionnaire historique des argots français, Paris, Larousse.

Fishman Joshua A., 1971 : Sociolinguistique, Paris-Bruxelles, Nathan-Labor.

Frei Henri, 1929 : La grammaire des fautes, Genève, Kundig.

GADET Françoise (dir.) [à paraître] : « Actualité des problématiques de la variation », Langages.

GaRdin Bernard et Marcellesi Jean-Baptiste (dir.), 1980 : Sociolinguistique, approches, théories, pratiques, Rouen et Paris, PUF.

Gillínon Jules et Edmont Edmond, 1902-1920 : Atlas Linguistique de la France, Paris, Champion, 17 vol. + 1 supplément.

GILliéron Jules et ROQUes Mario, 1912 : Etudes de géographie linguistique, Paris, Champion. 
Gilliéron Jules, 1918 : Généalogie des mots désignant l'abeille, Paris, Champion. GOODY Jack, 1977 : La raison graphique. La domestication de la pensée sauvage, Paris, Minuit.

GumperZ J.A. et Hymes Dell (dir.), 1964 : « The ethnography of communication ", American Anthropologist, 66, 2 (hors série).

Jaubert Hippolyte-François, 1855-1858 : Glossaire du Centre de la France, Paris, Chaix ( 2 vol.).

KUKENHEIM Louis, 1966 : Esquisse historique de la linguistique française, Leyde, Universitaire Pers Leiden.

Labov William, 1976 : Sociolinguistique, Paris, Minuit.

LABOV William, 1979 : Le parler ordinaire, Paris, Minuit, 2 vol.

LEROND Alain (dir.), 1973 : « Les parlers régionaux », Langue Française, 18, mai 1973.

Levi-Strauss Claude, 1955 : Tristes tropiques, Paris, Plon.

MARCELlesI Jean-Baptiste (dir.), 1971 : « Linguistique et société », Langue Française, 9, février 1971.

Martinet André, 1945 : La prononciation du français contemporain, Paris, Droz.

Mistral Frédéric, 1932 : Tresor dou Felibrige, Paris, Dalagrave ( ${ }^{\text {re }}$ édition en deux volumes : 1878-1886).

Millardet Georges, 1910 : Petit atlas linguistique d'une région des Landes. Contribution à la dialectologie gasconne, Toulouse, Privat.

Millardet Georges, 1923 : Linguistique et dialectologie romanes. Problèmes et méthodes, Montpellier, SELR.

Monfrin Jacques, 1978 : Honoré Champion et sa librairie, Paris, Champion.

Montesson Charles-Raoul de, 1859 : Vocabulaire du Haut-Maine, Paris-Le Mans.

Nizier du Puitspelu - pseudonyme de Clair Tisseur - (1890), Dictionnaire étymologique du patois lyonnais, Lyon, Georg.

Paris Gaston, 1893 : «Les parlers de France », in Bulletin de la Société des Parlers de France, I, 1, pp. 1-19.

Paris Gaston, Tobler Adolf, Meyer-Lubke, Thomas Antoine, Roques Mario, 1903 : Notice sur l'Atlas Linguistique de la France, Paris, Champion.

PAssy Paul, 1906, Les sons du français : leur formation, leur combinaison, leur représentation, Paris, Didot, 1906 ( ${ }^{\text {er }}$ édition : 1887).

Pernot Hubert, 1928 : «L'Institut de Phonétique de l'Université de Paris », in Revue de Phonétique, V, 1, pp. 1-15.

Picoche Jacqueline, 1973 : « Les monographies dialectales (domaine galloroman) ", in Lerond (1973), pp. 8-41.

Pop Sever, 1950 : La Dialectologie. Aperçu historique et méthodes d'enquêtes linguistiques. I. Dialectologie romane, Gembloux, Duculot.

Pop Sever, 1955 : Bibliographie des questionnaires linguistiques, Louvain, Commission d'Enquête Linguistique du CIPL.

RonJAT Jules, 1930 : Grammaire istorique des parlers provençaux modernes, SELR, Montpellier.

Saussure Ferdinand de, 1972 : Cours de linguistique générale, Paris, Payot (édition critique établie par Tullio de Mauro). 
SEgUY Jean, 1973 : « Les Atlas linguistiques de la France par régions », in Lerond (1973), pp. 65-90.

SOPTRAJANOV Georgi, 1978 : Bibliographie analytique de la linguistique française, Genève, Slatkine.

SumpF Joseph (dir.), 1968 : «Sociolinguistique », Langages, 11.

TERRACHER A.-L., 1914 : Les aires morphologiques dans les parlers du nord-ouest de l'Angoumois (1800-1900), Paris, Champion.

Tourtoulon Charles de et Bringuier Octave, 1876: « Etude sur les limites géographiques de la langue d'oc et de la langue d'oil ", in Archives des missions, Troisième série, tome III.

Tourtoulon Charles de, 1890 : Des dialectes, de leur classification et de leur délimitation géographique, Paris, Maisonneuve.

Wagner Robert-Léon, 1967 : «Le français », Revue de l'Enseignement Supérieur, 3-4, pp. 11-22.

Wartburg Walther von, Keller Hans-Erich et GeulJans Robert, 1969 : Bibliographie des Dictionnaires Patois Gallo-Romans (1550-1967), Genève, Droz.

Weinreich Uriel, 1953 : Languages in Contact, La Haye, Mouton.

Willems Dominique (dir.), 1990 : Travaux de Linguistique, 21.

XXX (Devigne Roger), 1935 : Le musée de la parole et du geste : les collections le laboratoire - la phonothèque, Paris, Musée de la Parole.

Bulletin de la Société des parlers de France (1893-1900), Paris, SPF.

Cahiers du français des années quatre-vingts (1985 $\rightarrow$ ), Saint-Cloud, CREDIF.

Langage et société $(1977 \rightarrow)$, Paris, MSH.

Recherches sur le Français Parlé $(1977 \rightarrow)$, Aix-en-Provence, Publications de l'Université.

Revue des langues romanes $(1870 \rightarrow)$, Montpellier, SELR.

Revue des patois (1887-1888), Paris.

Revue des patois gallo-romans (1887-1893), Paris, Champion puis Welter.

Romania (1872 $\rightarrow$ ), Paris, Champion. 